\title{
Covers, orientations and factors
}

\author{
Péter Csikvári* \\ MTA-ELTE Geometric and Algebraic Combinatorics Research Group \& \\ ELTE: Eötvös Loránd University \\ Mathematics Institute, Department of Computer Science \\ H-1117 Budapest, Pázmány Péter sétány 1/C, Hungary \\ peter.csikvari@gmail.com \\ András Imolay ${ }^{\dagger}$ \\ ELTE: Eötvös Loránd University \\ H-1117 Budapest, Pázmány Péter sétány 1/C, Hungary \\ imolay.andras@gmail.com
}

Submitted: May 27, 2019; Accepted: Jul 2, 2020; Published: Aug 7, 2020

(C) The authors. Released under the CC BY-ND license (International 4.0).

\begin{abstract}
Given a graph $G$ with only even degrees, let $\varepsilon(G)$ denote the number of Eulerian orientations, and let $h(G)$ denote the number of half graphs, that is, subgraphs $F$ such that $d_{F}(v)=d_{G}(v) / 2$ for each vertex $v$. Recently, Borbényi and Csikvári proved that $\varepsilon(G) \geqslant h(G)$ holds true for all Eulerian graphs, with equality if and only if $G$ is bipartite. In this paper we give a simple new proof of this fact, and we give identities and inequalities for the number of Eulerian orientations and half graphs of a 2-cover of a graph $G$.
\end{abstract}

Mathematics Subject Classifications: 05C30, 05C70, 05C76, 05C45

\section{Introduction}

In this paper we study the number of orientations and factors of Eulerian graphs. Recall that a graph $G$ is Eulerian if every vertex of $G$ has even degree. In the literature it is

*The first author was supported by the Marie Skłodowska-Curie Individual Fellowship grant no. 747430, and before that grant he was partially supported by the Hungarian National Research, Development and Innovation Office, NKFIH grant K109684 and Slovenian-Hungarian grant NN114614, and by the ERC Consolidator Grant 648017.

${ }^{\dagger}$ The second author is partially supported by the New National Excellence Program (ÚNKP) and by the European Union, co-financed by the European Social Fund (EFOP-3.6.3-VEKOP-16-2017-00002). 
often assumed that an Eulerian graph is also connected, but we will not require connectedness in this paper. Let $\varepsilon(G)$ denote the number of Eulerian orientations, that is, the orientations where every vertex has in-degree equal to the out-degree. Counting Eulerian orientations has triggered considerable interest in combinatorics, computer science and statistical physics. Probably, the best known result is due to Lieb [11] who determined the asymptotic number of Eulerian orientations of large grid graphs. Schrijver [15] gave a lower bound on the number of Eulerian orientations in terms of the degree sequence. Welsh [18] observed that for a 4-regular graph the Tutte-polynomial evaluation $\left|T_{G}(0,-2)\right|$ is exactly the number of Eulerian orientations since nowhere-zero $Z_{3}$-flows and Eulerian orientations are in one-to-one correspondence for 4-regular graphs. Mihail and Winkler [13] gave an efficient randomized algorithm to sample and approximately count Eulerian orientations.

Let $h(G)$ denote the number of half graphs, that is, subgraphs $F$ satisfying that $d_{F}(v)=d_{G}(v) / 2$ for every vertex $v$. Note that $h(G)>0$ if $G$ is not only Eulerian, but each of its connected component has an even number of edges. This condition is clearly necessary to have a half graph, and also sufficient: every second edge of an Eulerian tour will determine a half graph.

Recently, Borbényi and Csikvári [4] proved that $\varepsilon(G) \geqslant h(G)$ holds true for all Eulerian graphs with equality if and and only if $G$ is bipartite. This inequality fits into a series of inequalities comparing the number of orientations and subgraphs with the same property. For instance, it is known that the number of acyclic orientations is less than or equal to the number of acyclic subgraphs, that is, forests. The paper of Kozma and Moran [10] contains many more such inequalities, and sometimes equalities. In this paper we give a simple new proof of the fact $\varepsilon(G) \geqslant h(G)$, and we give identities and inequalities for the number of Eulerian orientations and half graphs of a 2-cover of a graph $G$. In fact, we study the number of orientations and factors of 2-covers with prescribed in-degree and degree sequences, respectively.

\subsection{Results.}

For an edge set $A \subseteq E(G)$ let $\varepsilon(A)$ denote the number of Eulerian orientations of the graph $(V, A)$. Similarly, let $h(A)$ denote the number of half graphs of the graph $(V, A)$. Our first result is an identity for $\varepsilon(G)$ and $h(G)$.

Theorem 1. Let $G$ be an Eulerian graph with edge set E. Then

$$
\varepsilon(G)^{2}=\sum_{A \subseteq E} \varepsilon(A) \varepsilon(E \backslash A)
$$

and

$$
h(G)^{2}=\sum_{A \subseteq E} h(A) h(E \backslash A) .
$$

Using the identities of Theorem 1 we can easily give a new proof of the following theorem of Borbényi and Csikvári [4]. 
Theorem 2 (Borbényi and Csikvári [4]). Let $G$ be an Eulerian graph. Then $\varepsilon(G) \geqslant h(G)$ with equality if and only if $G$ is bipartite.

Note that for a bipartite graph $G=(A, B, E)$ it is trivial that $\varepsilon(G)=h(G)$ since there is a natural correspondence between subgraphs and orientations: if an edge is oriented towards $B$, then put it into the subgraph, and if it is oriented towards $A$, then do not include it into the subgraph. This gives also a bijection between Eulerian orientations and half graphs.

As we will see it is natural to consider the number of Eulerian orientations and half graphs of 2-covers of an Eulerian graph $G$.

Definition 3. A $k$-cover (or $k$-lift) $H$ of a graph $G$ is defined as follows. The vertex set of $H$ is $V(H)=V(G) \times\{0,1, \ldots, k-1\}$, and if $(u, v) \in E(G)$, then we choose a perfect matching between the vertices $(u, i)$ and $(v, j)$ for $0 \leqslant i, j \leqslant k-1$. If $(u, v) \notin E(G)$, then there are no edges between $(u, i)$ and $(v, j)$ for $0 \leqslant i, j \leqslant k-1$.
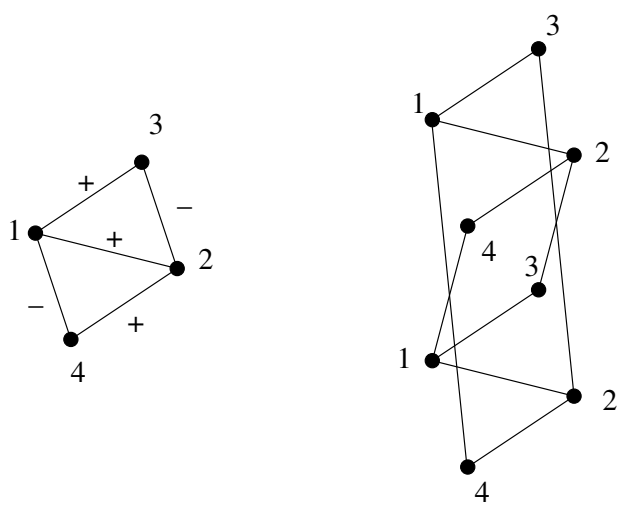

Figure 1: A 2-lift.

When $k=2$ one can encode the 2-lift $H$ by putting signs on the edges of the graph $G$ : the + sign means that we use the matching $((u, 0),(v, 0)),((u, 1),(v, 1))$ at the edge $(u, v)$, the - sign means that we use the matching $((u, 0),(v, 1)),((u, 1),(v, 0))$ at the edge $(u, v)$. For instance, if we put + signs to every edge, then we simply get $G \cup G$ as $H$, and if we put - signs everywhere, then the obtained 2-cover $H$ is simply $G \times K_{2}$. (In general, the product graph $G \times H$ is defined as follows: $V(G \times H)=V(G) \times V(H)$ and the vertices $\left(u_{1}, v_{1}\right)$ and $\left(u_{2}, v_{2}\right)$ are adjacent if and only if $\left(u_{1}, u_{2}\right) \in E(G)$ and $\left(v_{1}, v_{2}\right) \in E(H)$.) Note that the graph $G \times K_{2}$ is bipartite, it is also called the bipartite double cover of $G$. Observe that if $G$ is bipartite, then $G \cup G=G \times K_{2}$, but other 2-covers might differ from $G \cup G$.

Graph cover techniques played important roles in the resolution of many open problems. Marcus, Spielmann and Srivastava [12] used graph covers to construct Ramanujan graphs. The idea was suggested by Bilu and Linial [3]. Zhao [20] used the bipartite double cover to prove a conjecture of Alon [2] and Kahn [9] on the number of independent sets. Later he developed his ideas in the paper [21]. Ruozzi [14] proved a conjecture 
of Sudderth, Wainwright, and Willsky [19] on the Bethe approximation of an attractive graphical model by building on an observation due to Vontobel [17] connecting graph covers with Bethe approximation. Csikvári [6] combined graph covers with graph limit theory to prove the so-called Lower Matching Conjecture of Friedland, Krop and Markström [7]. The properties of random lifts are also widely studied, see for instance the papers [1] and [8].

Theorem 4. Let $G$ be an Eulerian graph with edge set E. Then

$$
\varepsilon\left(G \times K_{2}\right)=h\left(G \times K_{2}\right)=\sum_{A \subseteq E} \varepsilon(A) h(E \backslash A) .
$$

Combining Theorem 4 with Theorem 2 we get that

$$
\varepsilon(G \cup G) \geqslant \varepsilon\left(G \times K_{2}\right)=h\left(G \times K_{2}\right) \geqslant h(G \cup G)
$$

since $\varepsilon(A) \geqslant h(A)$ and $\varepsilon(E \backslash A) \geqslant h(E \backslash A)$, and the equality holds true since $G \times K_{2}$ is a bipartite graph. This gives a slight refinement of Theorem 2 that already gave $\varepsilon(G \cup G) \geqslant h(G \cup G)$ : we can see that we can put $\varepsilon\left(G \times K_{2}\right)$ between them.

These inequalities can be generalized as follows. Let $H$ be an arbitrary 2-cover of an Eulerian graph $G$. Then

$$
\varepsilon(G \cup G) \geqslant \varepsilon(H) \text { and } h\left(G \times K_{2}\right) \geqslant h(H) .
$$

In fact, even more general statement is true. To spell out this generalization we need the concepts $\varepsilon_{\underline{r}}(G)$ and $h_{\underline{r}}(G)$.

Definition 5. Let $\underline{r}^{G}=\left(r_{v}\right)_{v \in V(G)} \in \mathbb{Z}^{V(G)}$. Let $\varepsilon_{\underline{r}}(G)$ denote the number of orientations of $G$ with in-degree $r_{v}$ at vertex $v$. We will call such an orientation an $\underline{r}$-orientation.

Similarly, let $h_{\underline{r}}(G)$ be the number of subgraphs $F$ of $G$ with degree $d_{F}(v)=r_{v}$ for each vertex $v$. We will call such a subgraph an $\underline{r}$-factor.

Clearly, if $r_{v}=d_{G}(v) / 2$ for each vertex $v$, then $\varepsilon_{\underline{r}}(G)=\varepsilon(G)$ and $h_{\underline{r}}(G)=h(G)$. Other notable case is when $r_{v}=r$ for all $v$, then $h_{\underline{r}}(G)$ counts the number of $r$-factors.

Definition 6. Let $\underline{r}^{G}=\left(r_{v}\right)_{v \in V(G)} \in \mathbb{Z}^{V(G)}$ and let $H$ be a $k$-cover of $G$. We say that $\underline{r}^{H}=\left(r_{v}\right)_{v \in V(H)} \in \mathbb{Z}^{V(H)}$ is induced by $\underline{r}^{G}$ if $r_{u^{\prime}}=r_{u}$ for all lifts $u^{\prime} \in V(H)$ of $u \in V(G)$.

In the following statements we can even drop the condition of $G$ being Eulerian.

Theorem 7. Let $\underline{r}^{G}=\left(r_{v}\right)_{v \in V(G)} \in \mathbb{Z}^{V(G)}$. Let $H$ be an arbitrary 2-cover of the graph $G$. Let us denote by $\underline{r}$ the induced vector of $\underline{r}^{G}$ on $H$ and $G \cup G$. Then

$$
\varepsilon_{\underline{r}}(G \cup G) \geqslant \varepsilon_{\underline{r}}(H) .
$$

In other words, for any vector $\underline{r} \in \mathbb{Z}^{V(G)}$, the 2 -cover $H$ that maximizes $\varepsilon_{\underline{r}}(H)$ is $G \cup G$. 
Theorem 8. Let $\underline{r}^{G}=\left(r_{v}\right)_{v \in V(G)} \in \mathbb{Z}^{V(G)}$. Let $H$ be an arbitrary 2-cover of a graph $G$. Let us denote by $\underline{r}$ the induced vector of $\underline{r}^{G}$ on $H$ and $G \times K_{2}$. Then

$$
h_{\underline{r}}\left(G \times K_{2}\right) \geqslant h_{\underline{r}}(H) .
$$

In other words, for any vector $\underline{r} \in \mathbb{Z}^{V(G)}$, the 2 -cover $H$ that maximizes $h_{\underline{r}}(H)$ is $G \times K_{2}$.

Note that Theorems 7 and 8 provide yet another proof of Theorem 2 by

$$
\varepsilon(G)^{2}=\varepsilon(G \cup G) \geqslant \varepsilon\left(G \times K_{2}\right)=h\left(G \times K_{2}\right) \geqslant h(G \cup G)=h(G)^{2},
$$

where in the equality $\varepsilon\left(G \times K_{2}\right)=h\left(G \times K_{2}\right)$ we use only the fact that $G \times K_{2}$ is a bipartite graph, so we only use the trivial part of Theorem 2 .

Next we generalize the concept of Eulerian orientations and half graphs. To every edge of $G$ let us assign either $o$ or $s$, that is, orientation or subgraph. Then for all edge we have two choices: if we assigned $o$ to the edge, then we need to orient it, so when we consider the in-degree, we choose one of the end points and add 1 to it, and add 0 to the other endpoint. If we assigned $s$ for the edge, then we need to decide whether we put this edge into subgraph or not, so we either add 1 to the degrees of both endpoints, or add 0 to the degrees of both endpoints. We will call such a configuration a factorientation. We will call the contribution of the edges to the vertex $v$ the mixed degree of $v$, that is, it is the sum of the in-degree from oriented edges and the degree coming from the subgraph. After we choose $o$ or $s$ for every edge, we say that a factorientation is balanced, if for every vertex $v$ the mixed degree is $d_{G}(v) / 2$. Let $g(G)$ be the number of balanced factorientations. We can see that this is a generalization of both Eulerian orientations and half graphs, because if we assign $o$ to each edge, then $g(G)=\varepsilon(G)$ and if we assign $s$ to each edge, then $g(G)=h(G)$.

Theorem 9. Let $G$ be a graph with each edge equipped with one of the 4 decorations $(+, o),(+, s),(-, o),(-, s)$. Let $H$ be a 2-cover of $G=(V, E)$ encoded by the + and signs. Furthermore, we put the decorations $o$ and $s$ to the edges of $H$ consistently with the decoration of $G$, that is, for each edge $e \in E(G)$ the 2-lifts of this edge get the same letter (o or $s$ ). For an edge subset $B \subseteq E(G)$ let $\bar{B}$ denote the graph with vertex set $V(G)$ and edge set $B$, where for each edge $e \in B$ with $a$ - sign on it we swap the decorations $o$ to $s$ and vice versa. Then the number of balanced factorientations with respect to these decorations satisfies

$$
g(H)=\sum_{A \subseteq E} g(A) g(\overline{E \backslash A}) .
$$

Clearly, Theorem 9 generalizes both Theorem 1 and 4. We can also generalize Theorem 2 as follows.

Theorem 10. Let $G$ be an Eulerian graph with decorations o and $s$ on the edges. Let $g(G)$ be the number of corresponding balanced factorientations. Then $g(G) \leqslant \varepsilon(G)$.

With the method that we use to prove Theorem 1 we can also prove another nice result about orientations:

Theorem 11. Let $G$ be an Eulerian graph. Then $\varepsilon_{\underline{r}}(G)$ is maximal if $r_{v}=d_{G}(v) / 2$ for all vertex $v$. 


\subsection{Organization of this paper and notations.}

In the next section we prove Theorems 1, 2 and 4. In Section 3 we prove Theorem 7 and Theorem 8. In Section 4 we prove Theorems 9 and 10. In Section 5 we prove Theorem 11. We end the paper with an open problem.

Notations. For a graph $G$ and a vertex $v$ let $E_{v}$ denote the edges incident to $v$ in the graph $G$ and let $d_{G}(v)=\left|E_{v}\right|$. Furthermore, $N_{G}(v)$ denotes the set of neighbors of $v$. For a graph $G=(V, E)$ let $\operatorname{pm}(G)$ denote the number of perfect matchings. If $H$ is a 2-cover of $G$, then $V_{0}$ and $V_{1}$ will denote the two copies of the vertex set. In particular, $G \times K_{2}$ is a bipartite graph with bipartite classes $V_{0}$ and $V_{1}$. For a vertex $u \in V(G)$ let $u_{0}$ and $u_{1}$ denote the two copies of $u$ in the 2-cover $H$.

\section{Proof of Theorems 1,2 and 4}

In this section we prove Theorems 1, 2 and 4.

Proof of Theorem 1. We only prove the identity $h(G)^{2}=\sum_{A \subseteq E} h(A) h(E \backslash A)$. The proof of the other identity is very similar. Consider $S, T \subseteq E(G)$ determining two half graphs. Let $A=S \Delta T$ be the symmetric difference of the half graphs $S$ and $T$. Let $B=E \backslash A$, then $B=(S \cap T) \cup(E \backslash(S \cup T))$. Recall that $E_{v}$ denotes the edges incident to the vertex $v$. Since $\left|S \cap E_{v}\right|=\left|T \cap E_{v}\right|=d_{G}(v) / 2$ we have $\left|E_{v} \cap(A \cap S)\right|=\left|E_{v} \cap(A \cap T)\right|$ and $\left|E_{v} \cap(S \cap T)\right|=\mid E_{v} \cap(E \backslash(S \cup T) \mid$. In other words, $A \cap S$ is a half graph of $A$, and $B \cap(S \cap T)$ is a half graph of $B$. Clearly, $S$ and $T$ determine $A, A \cap S, B, B \cap(S \cap T)$. But it is also true that $A, A \cap S, B, B \cap(S \cap T)$ determine $S$ and $T$ since $S=(A \cap S) \cup(S \cap T)$ and $T=(A \cap T) \cup(S \cap T)$. The number of quadruple $A, A \cap S, B, B \cap(S \cap T)$ is clearly $\sum_{A \subseteq E} h(A) h(E \backslash A)$. Hence

$$
h(G)^{2}=\sum_{A \subseteq E} h(A) h(E \backslash A) .
$$

In case of Eulerian orientations let $S$ and $T$ be two Eulerian orientations, and let $A$ be the set of edges where the orientations coincide and $B=E \backslash A$ be the remaining edges. Similarly to the previous discussion $S$ restricted to $A$ and $B$ gives an Eulerian orientation. The rest of the proof is the same.

Proof of Theorem 4. The proof is very similar to the proof of Theorem 1. Since $G \times$ $K_{2}=\left(V_{0}, V_{1}, E\right)$ is bipartite we have $\varepsilon\left(G \times K_{2}\right)=h\left(G \times K_{2}\right)$. Consider a half graph $S$ of $G \times K_{2}$. Let $\pi: G \times K_{2} \rightarrow G$ be the natural projection. For $k=0,1,2$ let $A_{k}=\left\{e \in E|| \pi^{-1}(e) \cap S \mid=k\right\}$. Since $S$ was a half graph of $G \times K_{2}$ we get that for each vertex $v$ we have $\left|E_{v} \cap A_{0}\right|=\left|E_{v} \cap A_{2}\right|$. In other words, $A_{0}$ is a half graph of $A_{0} \cup A_{2}$. Let us orient an edge $(u, v) \in A_{1}$ from $u$ to $v$ if $\left(u_{0}, v_{1}\right) \in S$. Since $S$ was a half graph of $G \times K_{2}$ this gives an Eulerian orientation of the edges of $A_{1}$. Clearly, from the Eulerian 
orientation of $A_{1}$ and the half graph $A_{0}$ of $A_{0} \cup A_{2}$ we can immediately reconstruct $S$. Hence

$$
h\left(G \times K_{2}\right)=\sum_{A_{1} \subseteq E} \varepsilon\left(A_{1}\right) h\left(A_{0} \cup A_{2}\right)=\sum_{A \subseteq E} \varepsilon(A) h(E \backslash A) .
$$

Proof of Theorem 2. We prove the statement by induction on the number of edges of $G$. If the graph has no edge, then the statement is trivial. If it consists of a single cycle of length $k$ together with isolated vertices, then $\varepsilon(G)=2$ and $h(G)=0$ or 2 depending on $k$ being odd or even. So in this case the theorem is true. If $G$ is different from a single cycle together with isolated vertices, then we use induction:

$$
\varepsilon(G)^{2}-2 \varepsilon(G)=\sum_{\substack{A \subseteq E \\ A \neq \emptyset, E}} \varepsilon(A) \varepsilon(E \backslash A) \geqslant \sum_{\substack{A \subseteq E \\ A \neq \emptyset, E}} h(A) h(E \backslash A)=h(G)^{2}-2 h(G) .
$$

Since $\varepsilon(G) \geqslant 1$ and the function $x^{2}-2 x$ is a monotone increasing function for $x \geqslant 1$ we get that $\varepsilon(G) \geqslant h(G)$.

In case of a bipartite graph we have equality by the bijection between subgraphs and orientations. If the graph $G$ is not bipartite, then it contains an odd cycle on an edge subset $A$ for which we have strict inequality $\varepsilon(A)>h(A)$. Then we have strict inequality in $\varepsilon(G)^{2}-2 \varepsilon(G)>h(G)^{2}-2 h(G)$ implying $\varepsilon(G)>h(G)$. Hence equality holds if and only if $G$ is bipartite.

\section{Proof of Theorems 7 and 8}

In this section we prove Theorems 7 and 8 . The proofs rely on three observations, one of them is due to Schrijver relating the number of $\underline{r}$-orientations of a graph $G$ to the number of perfect matchings of a certain bipartite graph $G^{*}$ constructed from $G$. (In fact, we will slightly modify it, but still attribute it to Schrijver.) A similar observation connects the number of $\underline{r}$-factors of a graph $G$ to the number of perfect matchings of another graph $G^{* *}$ constructed from $G$. The last observation is due to Csikvári and gives an inequality between the number of perfect matchings of certain 2-covers of a graph $G$.

Lemma 12 (Schrijver [15]). Let $G$ be a graph, and let $G^{*}$ be the following bipartite graph. On one side of the bipartite graph every vertex corresponds to an edge $e \in E(G)$. On the other side of the bipartite graph we take $r_{v}$ copies of each vertex $v$. Finally, an edge $e=(u, v)$ is adjacent to all copies of $u$ and $v$. Then

$$
\operatorname{pm}\left(G^{*}\right)=\varepsilon_{\underline{r}}(G) \prod_{v \in V(G)} r_{v} !
$$

Proof. Let $R=\prod_{v \in V(G)} r_{v}$ !. There is an 1 to $R$ map from the set of $\underline{r}$-orientations to the set of perfect matchings of $G^{*}$. Namely, if the edges $\left(u, v_{i}\right) \in E(G)$ for $i=1, \ldots r_{u}$ are oriented towards $u$, then we take the union of perfect matchings between $e_{u v_{i}}$ and the $r_{u}$ copies of $u$ to get a perfect matching of $G^{*}$. 
A similar lemma enable us to encode $h_{\underline{r}}$ via perfect matchings. A qualitative version of this lemma appeared in [16].

Lemma 13 (Tutte [16]). Let $G$ be a graph, and let $G^{* *}$ be the following graph. For each edge $e=(u, v)$ we introduce two vertices $e_{u v}$ and $e_{v u}$, and for each vertex $v$ we introduce $r_{v}$ copies of $v$. Then we connect $e_{u v}$ with $e_{v u}$, and we also connect the $r_{u}$ copies of $u$ with $e_{v u}$ for each $v \in N_{G}(u)$. Then

$$
\operatorname{pm}\left(G^{* *}\right)=h_{\underline{r}}(G) \prod_{v \in V(G)} r_{v} !
$$

Proof. Let $R=\prod_{v \in V(G)} r_{v}$ !. Again there is an 1 to $R$ map from the set of $\underline{r}$-factors to the set of perfect matchings of $G^{* *}$. Namely, if the edges $\left(u, v_{i}\right) \in E(G)$ for $i=1, \ldots r_{u}$ are in the $\underline{r}$-factor, then to get a perfect matching of $G^{* *}$, we take the union of perfect matchings between $e_{v_{i} u}$ and the $r_{u}$ copies of $u$ together with those edges $\left(e_{x y}, e_{y x}\right)$ for which $(x, y) \in E(G)$, but is not in the $\underline{r}$-factor.

Next we need a lemma that relates perfect matchings with covers.

Lemma 14 (Csikvári $[6,5]$ ). Let $G$ be a graph, and let $H$ be an arbitrary 2-cover of $G$. Then

$$
\operatorname{pm}(H) \leqslant \operatorname{pm}\left(G \times K_{2}\right) .
$$

In particular, if $G$ is bipartite, then $\operatorname{pm}(H) \leqslant \operatorname{pm}(G)^{2}$.

Sketch of the proof. Let us project the edges of a perfect matching of a 2-cover $H$ to the graph $G$. The obtained configuration consists of cycles and double-edges, that is, two edges projected to the same edge. (Every degree must be 2 in the obtained configuration.) For such a configuration we can count the number of preimages. Each cycle can be lifted in at most 2 ways since the preimage of one edge determines the preimage of the subsequent edges in the cycle. It may occur though that we cannot close the cycle. (This happens for instance if try to lift a 3-cycle in the union of two 3-cycles.) On the other hand, it is easy to see that on $G \times K_{2}$ every cycle can be lifted in exactly 2 ways. This means that every configuration has at least as many preimages on $G \times K_{2}$ than on another 2-cover $H$.

Proof of Theorem 7. Let $H$ be an arbitrary cover of the graph $G$. Let us construct the bipartite graphs $G^{*}$ and $H^{*}$ of Lemma 12 . Observe that $H^{*}$ is also a 2-cover of $G^{*}$, and so by Lemma 14 we have $\operatorname{pm}\left(G^{*}\right)^{2} \geqslant \operatorname{pm}\left(H^{*}\right)$. Using Lemma 12 we have

$$
\operatorname{pm}\left(G^{*}\right)^{2}=\varepsilon_{\underline{r}}(G)^{2}\left(\prod_{v \in V(G)} r_{v} !\right)^{2}
$$

and

$$
\operatorname{pm}\left(H^{*}\right)=\varepsilon_{\underline{r}}(H) \prod_{v \in V(H)} r_{v} !=\varepsilon_{\underline{r}}(H)\left(\prod_{v \in V(G)} r_{v} !\right)^{2} .
$$

Hence $\varepsilon_{\underline{r}}(G)^{2} \geqslant \varepsilon_{\underline{r}}(H)$. 
Remark 15. An interesting application of Theorem 7 is the following. Let $T_{n, m}$ be the toroidal grid of size $n \times m$, that is, a grid of size $n \times m$ closed in a toroidal way to make it 4-regular. Then $\varepsilon\left(T_{n, m}\right) \geqslant\left(\frac{4}{3}\right)^{3 n m / 2}$. This can can be seen as follows. Lieb [11] showed that $\lim _{n, m \rightarrow \infty} \varepsilon\left(T_{n, m}\right)^{1 / n m}=\left(\frac{4}{3}\right)^{3 / 2}$. On the other hand, $T_{2 n, m}$ and $T_{n, 2 m}$ are 2-covers of $T_{n, m}$ so $\varepsilon\left(T_{n, m}\right)^{2} \geqslant \varepsilon\left(T_{2 n, m}\right)$ and $\varepsilon\left(T_{n, 2 m}\right)$ so it is necessary that $\varepsilon\left(T_{n, m}\right)^{1 / n m} \geqslant\left(\frac{4}{3}\right)^{3 / 2}$ for every $n, m$.

Proof of Theorem 8. Let $H$ be an arbitrary cover of the graph $G$. Let us construct the graphs $G^{* *}$ and $H^{* *}$ of Lemma 13. Observe that $H^{* *}$ is also a 2-cover of $G^{* *}$, and $(G \times$ $\left.K_{2}\right)^{* *}=G^{* *} \times K_{2}$ and so by Lemma 14 we have $\operatorname{pm}\left(\left(G \times K_{2}\right)^{* *}\right) \geqslant \operatorname{pm}\left(H^{* *}\right)$. Using Lemma 13 we have

$$
\operatorname{pm}\left(\left(G \times K_{2}\right)^{* *}\right)=h_{\underline{r}}\left(G \times K_{2}\right) \prod_{v \in V\left(G \times K_{2}\right)} r_{v} !=h_{\underline{r}}\left(G \times K_{2}\right)\left(\prod_{v \in V(G)} r_{v} !\right)^{2}
$$

and

$$
\operatorname{pm}\left(H^{* *}\right)=h_{\underline{r}}(H) \prod_{v \in V(H)} r_{v} !=h_{\underline{r}}(H)\left(\prod_{v \in V(G)} r_{v} !\right)^{2} .
$$

Hence $h_{\underline{r}}\left(G \times K_{2}\right) \geqslant h_{\underline{r}}(H)$.

\section{General 2-cover}

In this section we prove Theorems 9 and 10 .

Proof of Theorem 9. Consider a balanced factorientation $S$ of the graph $H$. Take the natural projection from $H$ to $G$, and let $A$ be the set of edges for which the projected edges coincide, that is, both edges are oriented in the same way if there is an $o$ on that edge, or they are both or neither in the subgraph if there is an $s$ on that edge.

Let $B=E \backslash A$. The factorientation $S$ was balanced so for all vertex $v$ the mixed degrees of $v_{0}$ and $v_{1}$ are both $d_{G}(v) / 2$. This means that after the natural projection - and the doubling of the original edges of the graph- the mixed degree of vertex $v$ is $d_{G}(v)$. If an edge is in $A$, then it contributes either 0 or 2 to the mixed degree of $v$, otherwise it contributes 1. Thus there must be equal number of 0's and 2's contributions, which means that if we restrict the graph to $A$, then we also get a balanced factorientation.

For an edge $(u, v) \in B$ if $(u, v)$ has a plus sign on it, then it contributes the same amount to the mixed degree of $u$ and $v$ as it contributed to the mixed degrees of $u_{0}$ and $v_{0}$ with the edge $\left(u_{0}, v_{0}\right)$ in $S$.

If $(u, v)$ has a minus sign on it, then in $(u, v)$ change $s$ to $o$ and vice verse. If it was $o$ before and the orientations were $u_{0} \rightarrow v_{1}$ and $v_{0} \rightarrow u_{1}$, then we do not put the edge $(u, v)$ into the subgraph. If it was $o$ before and the orientations were $u_{1} \rightarrow v_{0}$ and $v_{1} \rightarrow u_{0}$, then we do put the edge $(u, v)$ into the subgraph. Note that the contribution of these edges 
to the mixed degree of a vertex $u$ is the same as the contribution of the original edges to the vertex $u_{0}$.

Similarly, if it was an $s$ before, and $\left(u_{0}, v_{1}\right)$ was in the subgraph, but $\left(u_{1}, v_{0}\right)$ was not, then orient the edge $(u, v)$ from $v$ to $u$. This way it is again true that the contribution of these edges to the mixed degree of a vertex $u$ is the same as the contribution of the original edges to the vertex $u_{0}$.

So for all $(u, v) \in B$ we made sure that the contribution of the orientation or factor to the mixed degree of a vertex $u$ is the same contribution as the original edges to the mixed degree of the vertex $u_{0}$. Finally, observe that since the mixed degree of $u_{0}$ and $u_{1}$ was the same, and the edges of $A$ contributed the same to the mixed degrees, it is necessary that the mixed degree contributed by the edges of $B$ to the vertex $u$ is exactly $d_{B}(u) / 2$. This means that the constructed factorientation is balanced if we restrict to the edges of $B$.

Finally, observe that if we get a balanced factorientation of $A$ and $\overline{E \backslash A}$ then we can easily get back the balanced factorientation of $H$. This finishes the proof.

Proof of Theorem 10. The proof is practically the same as the proof of Theorem 2. We use induction to the number of edges. We have

$$
g(G)^{2}=g(G \cup G)=\sum_{A \subseteq E} g(A) g(\overline{E \backslash A})=\sum_{A \subseteq E} g(A) g(E \backslash A)
$$

since $G \cup G$ corresponds to the 2-cover with only + signs. By induction we have

$g(A) \leqslant \varepsilon(A)$ if $A \neq E$. Hence $g(G)^{2}-2 g(G) \leqslant \varepsilon(G)^{2}-2 \varepsilon(G)$ which implies that $g(G) \leqslant \varepsilon(G)$.

\section{$5 \quad$ Proof of Theorem 11}

In this section we prove Theorem 11.

Proof of Theorem 11. We prove the statement by induction on the number of edges. The statement is true for cycles.

Let $\underline{r} \in \mathbb{Z}^{V(G)}$ be an arbitrary vector and let $\underline{d} \in \mathbb{Z}^{V(G)}$ be the vector where $d_{v}=d_{G}(v)$. Notice that $\varepsilon_{\underline{r}}(G)=\varepsilon_{\underline{d}-\underline{r}}(G)$, because if we change the direction of all edges in an $\underline{r}$ orientation, then we get a $(\underline{d}-\underline{r})$-orientation. Consider $S$ and $T$ determining an $\underline{r}-$ orientation and a $(\underline{d}-\underline{r})$-orientation, respectively. Let $A \subseteq E$ be the set of edges where the two orientations coincide, and let $B=E \backslash A$ be the remaining edges. We claim that $A \cap S$ is an Eulerian orientation of $A$. To see this consider a vertex $v$ of $G$. Let $d_{S}^{A}(v)$ be the in-degree of $v$ in the orientation $S$ restricted to the set $A$. We similarly define $d_{S}^{B}(v)$, $d_{T}^{A}(v)$ and $d_{T}^{B}(v)$. Finally, let $d^{A}(v)$ be the degree of $v$ in the graph $(V, A)$. We similarly define $d^{B}(v)$. Then the in-degree of $v$ in the orientation $S$ is $r_{v}=d_{S}^{A}(v)+d_{S}^{B}(v)$. The in-degree of $v$ in the orientation $T$ is $d_{v}-r_{v}=d_{T}^{A}(v)+d_{T}^{B}(v)$. By the definition of $A$ we have $d_{S}^{A}(v)=d_{T}^{A}(v)$. By the definition of $B$ we have $d_{S}^{B}(v)=d^{B}(v)-d_{T}^{B}(v)$. Hence

$$
d^{A}(v)=d_{v}-d^{B}(v)
$$




$$
\begin{aligned}
& =r_{v}+\left(d_{v}-r_{v}\right)-d^{B}(v) \\
& =d_{S}^{A}(v)+d_{S}^{B}(v)+d_{T}^{A}(v)+d_{T}^{B}(v)-d^{B}(v) \\
& =d_{S}^{A}(v)+d_{T}^{A}(v)=2 d_{S}^{A}(v) .
\end{aligned}
$$

Since this is true for all vertex $v$ of $G$, we get that $A \cap S$ is an Eulerian orientation of $A$. From this it is easy to see that $S \cap B$ is an $\left(\underline{r}-\underline{d_{A}} / 2\right)$-orientation of $B$. If we choose $S \cap B$ that determines $T \cap B$, thus we get that

$$
\varepsilon_{\underline{r}}(G) \varepsilon_{\underline{d}-\underline{r}}(G)=\sum_{A \subseteq E} \varepsilon(A) \varepsilon_{\underline{r}-\underline{d_{A}} / 2}(E \backslash A)
$$

The degree of a vertex $v$ in $E \backslash A$ is $d_{G}(v)-d_{A}(v)$ so from induction we know that $\varepsilon_{\underline{r}-\underline{d_{A}} / 2}(E \backslash A) \leqslant \varepsilon_{\underline{d} / 2-\underline{d_{A}} / 2}(E \backslash A)$ if $A \neq \emptyset$. Thus

$$
\begin{aligned}
\varepsilon_{\underline{r}}(G) \varepsilon_{\underline{d}-\underline{r}}(G)-\varepsilon_{\underline{r}}(G) & =\sum_{\substack{A \subseteq E \\
A \neq \emptyset}} \varepsilon(A) \varepsilon_{\underline{r}-\underline{d_{A}} / 2}(E \backslash A) \\
& \leqslant \sum_{\substack{A \subseteq E \\
A \neq \emptyset \emptyset}} \varepsilon(A) \varepsilon_{\underline{d} / 2-\underline{d_{A}} / 2}(E \backslash A) \\
& =\varepsilon_{\underline{d} / 2}(G)^{2}-\varepsilon_{\underline{d} / 2}(G) .
\end{aligned}
$$

In the last step we used Theorem 1 . Recall that $\varepsilon_{\underline{r}}(G)=\varepsilon_{\underline{d}-\underline{r}}(G)$. Hence $\varepsilon_{\underline{r}}(G)^{2}-\varepsilon_{\underline{r}}(G) \leqslant$ $\varepsilon_{\underline{d} / 2}(G)^{2}-\varepsilon_{\underline{d} / 2}(G)$. This implies that $\varepsilon_{\underline{r}}(G) \leqslant \varepsilon_{\underline{d} / 2}(G)=\bar{\varepsilon}(\bar{G})$.

\section{Open problem}

We end this paper with an open problem.

Conjecture 16. Let $G$ be an Eulerian graph, and let $H$ be a $k$-cover of $G$. Then $\varepsilon(G)^{k} \geqslant \varepsilon(H)$.

\section{Acknowledgments}

The first author thanks László Kozma for calling attention to the paper [10]. The authors also thank the reviewers for helpful comments.

\section{References}

[1] N. Agarwal, K. Chandrasekaran, A. Kolla, and V. Madan, On the expansion of group-based lifts, SIAM Journal on Discrete Mathematics, 33:1338-1373, 2019.

[2] N. Alon, Independent sets in regular graphs and sum-free subsets of finite groups, Israel Journal of Mathematics, 73:247-256, 1991.

[3] Y. Bilu and N. Linial Lifts, discrepancy and nearly optimal spectral gap, Combinatorica, 26:495-519, 2006. 
[4] M. Borbényi and P. Csikvári, Counting degree-constrained subgraphs and orientations, Discrete Mathematics, 343:111842, 2020.

[5] P. Csikvári, Extremal regular graphs: the case of the infinite regular tree, arXiv:1612.01295, 2016.

[6] P. Csikvári, Lower matching conjecture, and a new proof of Schrijver's and Gurvits's theorems, Journal of the European Mathematical Society, 19:1811-1844, 2017.

[7] S. Friedland, E. Krop, and K. Markström, On the number of matchings in regular graphs, The Electronic Journal of Combinatorics, 15:\#R110, 2008.

[8] C. Greenhill, S. Janson, and A. Ruciński, On the number of perfect matchings in random lifts, Combinatorics, Probability and Computing, 19:791-817, 2010.

[9] J. Kahn, An entropy approach to the hard-core model on bipartite graphs, Combinatorics, Probability and Computing, 10:219-237, 2001.

[10] L. Kozma and S. Moran, Shattering, graph orientations, and connectivity, The Electronic Journal of Combinatorics, 20(3):\#P44, 2013.

[11] E. H. Lieb, Residual entropy of square ice, in Condensed Matter Physics and Exactly Soluble Models, Springer, 461-471, 2004.

[12] A. Marcus, D. A. Spielman, and N. Srivastava, Interlacing families I: Bipartite Ramanujan graphs of all degrees, Annals of Mathematics, 182:307-325, 2015.

[13] M. Mihail and P. Winkler, On the number of Eularian orientations ofa graph, in Proceedings of the third annual ACM-SIAM symposium on Discrete algorithms, Society for Industrial and Applied Mathematics, 138-145, 1992.

[14] N. Ruozzi, The Bethe partition function of log-supermodular graphical models, in Advances in Neural Information Processing Systems, 117-125, 2012.

[15] A. Schrijver, Bounds on the number of Eulerian orientations, Combinatorica, 3:375380, 1983.

[16] W. T. Tutte, A short proof of the factor theorem for finite graphs, Canadian Journal of Mathematics, 6:347-352, 1954.

[17] P. O. Vontobel, Counting in graph covers: A combinatorial characterization of the Bethe entropy function, IEEE Transactions on Information Theory, 59:6018-6048, 2013.

[18] D. Welsh, The Tutte polynomial, Random Structures 86 Algorithms, 15:210-228, 1999.

[19] A. S. Willsky, E. B. Sudderth, and M. J. Wainwright, Loop series and Bethe variational bounds in attractive graphical models, in Advances in neural information processing systems, 1425-1432, 2008.

[20] Y. Zhao, The number of independent sets in a regular graph, Combinatorics, Probability and Computing, 19:315-320, 2010.

[21] Y. Zhao The bipartite swapping trick on graph homomorphisms, SIAM Journal on Discrete Mathematics, 25:660-680, 2011. 